\title{
Autosomal dominant cone-rod dystrophy with negative electroretinogram
}

Department of

Ophthalmology,

Tohoku University,

School of Medicine,

Sendai, Japan

N Fujii

T Shiono

Y Wada

M Nakazawa

M Tamai

Yamada Eye Clinic, Yokote, Japan N Yamada

Correspondence to: T Shiono, MD, Departmen of Ophthalmology, Tohoku University School of Medicine, 1-1 Seiryo-machi, Aoba-ku, Sendai 980, Japan.

Accepted for publication 15 June 1995

Naotaka Fujii, Takashi Shiono, Yuko Wada, Mitsuru Nakazawa, Makoto Tamai, Nariyuki Yamada

\begin{abstract}
Aims-The negative electroretinogram (ERG) is observed in many hereditary retinal disorders. However, no reports have described a negative ERG in a family with autosomal dominant cone-rod dystrophy. A Japanese family with autosomal dominant cone-rod dystrophy with negative ERG is described.

Method-Members of a Japanese family with autosomal dominant cone-rod dystrophy were examined and evaluated with Goldmann and Humphrey perimetry, bright flash ERG with an intense white stimulus, rod, cone, and flicker ERGs, and fluorescein angiography. Molecular analysis of the rhodopsin and peripherin/RDS genes in the patients was also performed.

Results-A 45-year-old Japanese man (proband) presented with decreased visual acuity. His fundi revealed bull's eye maculopathy and his single flash bright ERG showed a negative configuration. Negative ERG responses also were found in his father, who had macular degeneration, and one of the proband's three children who showed no fundus changes. No irregularities were found in their rhodopsin or peripherin/RDS genes.

Conclusion-The condition of this family is believed to represent a previously undescribed autosomal dominant conerod dystrophy.

(Br f Ophthalmol 1995; 79: 916-921)
\end{abstract}

Many hereditary retinochoroidal disorders include macular lesions. Bull's eye maculopathy, for example, is not pathognomonic for a specific disorder, and it occurs in several hereditary retinal disorders, such as cone dystrophy, ${ }^{1}$ Stargardt's disease, ${ }^{2}$ retinitis pigmentosa, ${ }^{3}$ benign concentric annular macular dystrophy, ${ }^{4}$ and Batten's disease. ${ }^{5}$ A single bright flash electroretinogram (ERG), which is commonly used to identify retinochoroidal diseases, consists of an a-wave, a b-wave, and oscillatory potentials. In a normal ERG response, the amplitude of the a-wave is smaller than that of the b-wave. When the a-wave is normal and the b-wave does not go beyond the isoelectric point, the ERG is considered to have an electronegative configuration, the so called negative ERG. Among hereditary retinal disorders, a negative ERG is most frequently associated with $\mathrm{X}$ linked juvenile retinoschisis, ${ }^{6}$ congenital stationary nightblindness, ${ }^{7}$ and retinitis pigmentosa. ${ }^{8}$
Two Japanese groups, Miyake and associates ${ }^{9}$ and Kato and Watanabe, ${ }^{10}$ described atypical patients who had bull's eye maculopathy and a negative ERG. Recently, Kellner and Foerster ${ }^{11}$ reported three male patients with cone dystrophies and one subject without symptoms or fundus abnormalities who showed a negative photopic ERG response. However, no reports, to our knowledge, have described autosomal dominant cone-rod dystrophy associated with a negative scotopic ERG response.

We describe a Japanese family that had autosomal dominant cone-rod dystrophy with negative ERG findings. We believe that the condition identified in this family represents a previously undescribed autosomal dominant cone-rod dystrophy.

\section{Patients and methods}

The proband (case 1) and his children were examined at the Department of Ophthalmology, Tohoku University. The proband's father (case 2) was examined at Yamada Eye Clinic. Goldmann perimetry was performed using targets V3, I4, and I3, and Humphrey perimetry was performed using program 10-2 and 30-2. A dark adaptation curve was measured using Goldmann-Weekes dark adaptometry. After the patients underwent light adaptation for 5 minutes with 3000 lux illumination, visual thresholds were measured in the dark. A white target of $11^{\circ}$ was presented at $15^{\circ}$ above the fovea. The ERGs were recorded with the use of portable equipment (ERG 50, Kowa, Japan) that housed a 25 joule xenon flash lamp for stimulus of the entire retina. A monopolar contact lens electrode with an artificial pupil of $10 \mathrm{~mm}$ was used. A reference electrode was affixed to the patient's forehead. The time constant was 0.3 second. The distance between the lamp and the eye was $30 \mathrm{~cm}$. The pupil was dilated with a combination of $0.5 \%$ tropicamide and $0.5 \%$ phenylephrine hydrochloride. A bright flash ERG was recorded with a single bright white light after 30 minutes of dark adaptation. A scotopic (rod) ERG was recorded with a single dim blue light (Wratten 47, and ND 2.0 filters) in the dark. After light adaptation with 200 lux in patient's eye position for 20 minutes, a photopic (cone) ERG was elicited with red stimulus (Wratten 29 filter). A $30 \mathrm{~Hz}$ red $(660 \mathrm{~nm})$ flicker ERG was recorded using portable equipment (PE200A, Tomei, Japan). ${ }^{12}$ The contact lens had a built in light emitting diode and recording electrode. After light adaptation for 20 minutes with a light 
Table 1 Results of bright flash ERGs



$\Sigma \mathrm{O}=$ summation of amplitudes of $\mathrm{O} 1, \mathrm{O} 2, \mathrm{O} 3$, and $\mathrm{O} 4$.

measuring 200 lux at the position of patient's eye, the $30 \mathrm{~Hz}$ red flicker ERG was recorded.

An analysis of rhodopsin and peripherin/RDS genes was performed, as previously described. ${ }^{13}$ High molecular weight DNAs were isolated from the patient's peripheral white blood cells. We synthesised pairs of oligonucleotide primers to amplify segments containing each exon of the rhodopsin and the peripherin/RDS gene using polymerase chain reaction (PCR). The amplified DNA sequences were screened for mutations using single strand conformation polymorphism analysis (SSCP).

\section{Results}

Results of bright flash ERG in 50 normal eyes are described in Table 1 and Figure 4. Results of rod, cone, and flicker ERGs in 80 normal eyes are also given in Table 2 and Figure 4. Results of ERGs and other examinations performed in each patient are described in Tables 1 and 2, Figure 4, and case reports.

Molecular analysis of the rhodopsin and the peripherin/RDS genes in the patients showed no irregularities.

\section{REPORT OF CASES}

\section{Case 1}

A 45-year-old Japanese man (II-3, proband; Fig 1) was referred to our clinic on 22 January 1992 , with a complaint of decreased visual acuity. At age 43, he had decreased visual acuity and poor colour vision. At that time, an ophthalmologist diagnosed his condition as macular disease. Since then, his visual acuity and colour vision have deteriorated further. $\mathrm{He}$ has not reported nightblindness.

On examination, the best corrected visual acuity was $20 / 25$ with a $-4 \cdot 0 \mathrm{D}$ sphere in the right eye and $20 / 30$ with a $-3 \cdot 25 \mathrm{D}$ sphere in the left eye. Slit-lamp examination revealed no abnormal findings in the anterior segment, lens, or vitreous in either eye. Fundus examination disclosed normal optic disc and vessels in both eyes. The macula of each eye showed a circular area of hypopigmented atrophic lesions of retinal pigment epithelium (Fig 2A and B). Fluorescein angiography demonstrated bull's eye maculopathy in both eyes (Fig 2C and D). Visual fields tested by Goldmann and Humphrey perimetry showed normal responses in the periphery and a relative central scotoma corresponding to the atrophic macular area in each eye (Fig 3). His colour vision was tritan by testing with panel D-15. A dark adapted bright flash ERG showed a normal a-wave and a reduced b-wave (negative ERG; Table 1 and Fig 4). Amplitudes of oscillatory potentials were reduced (Table 1 and Fig 4). The rod ERG b-wave showed reduced amplitude with a delayed implicit time (Table 2 and Fig 4). The cone ERG showed reduced amplitude (Table 2 and Fig 4). Low responses with a delayed implicit time were found in the $30 \mathrm{~Hz}$ red flicker ERG (Table 2 and Fig 4). An electro-oculogram (EOG) was normal in both eyes (light peak/dark trough ratios were $2 \cdot 1$ in the right eye and 2.0 in the left eye). Dark adaptation findings by Goldmann-Weekes dark adaptometry showed an absent cone plateau, with a rapid fall to the normal final threshold $(2 \cdot 2 \log$ units). The patient had no history of taking chloroquine. His general physical condition was good. Plasma levels of cyclic AMP and cyclic GMP were 11.0 $\mathrm{pmol} / \mathrm{ml}$ and $2.8 \mathrm{pmol} / \mathrm{ml}$, respectively (normal range of cyclic AMP and cyclic GMP are $15-37 \mathrm{pmol} / \mathrm{ml}$ and $2-10 \mathrm{pmol} / \mathrm{ml}$, respectively).

\section{Case 2}

The 70-year-old father of the proband (I-1; Fig 1) complained of decreased visual acuity at age 50 and was told he had bilateral macular degeneration. His visual acuity deteriorated further and he was evaluated at age 70 by one of us (NY). His general health was good. The visual acuity was $20 / 400$ without correction in each eye. He read only the test plate with the Ishihara series. Biomicroscopy disclosed bilateral senile cataract and otherwise normal anterior segments and anterior vitreous.

Ophthalmoscopic findings included atrophy of the macular retinal pigment epithelium and choroid with normal discs and vessels (Fig 5). The bright flash ERG produced a negatively shaped response with reduced oscillatory potentials (Table 1 and Fig 4). Fluorescein

Table 2 Results of scotopic, photopic, and flicker ERGs

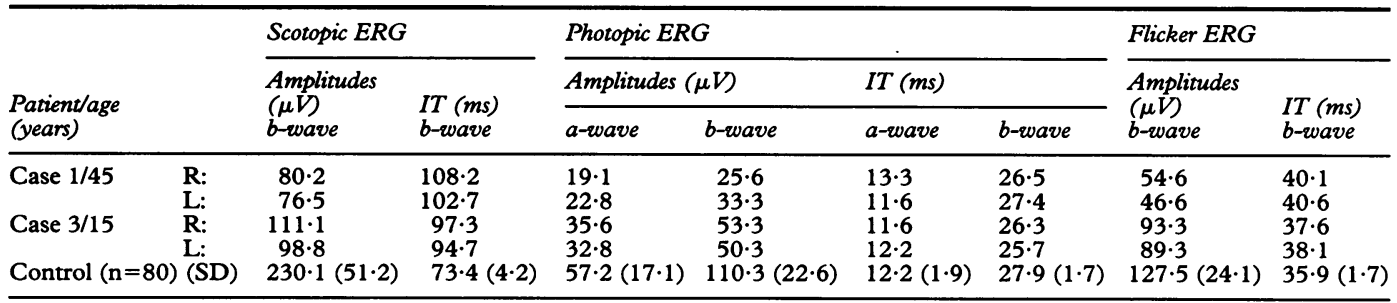

$\mathbf{I T}=$ implicit time; $\mathrm{ms}=$ milliseconds. 


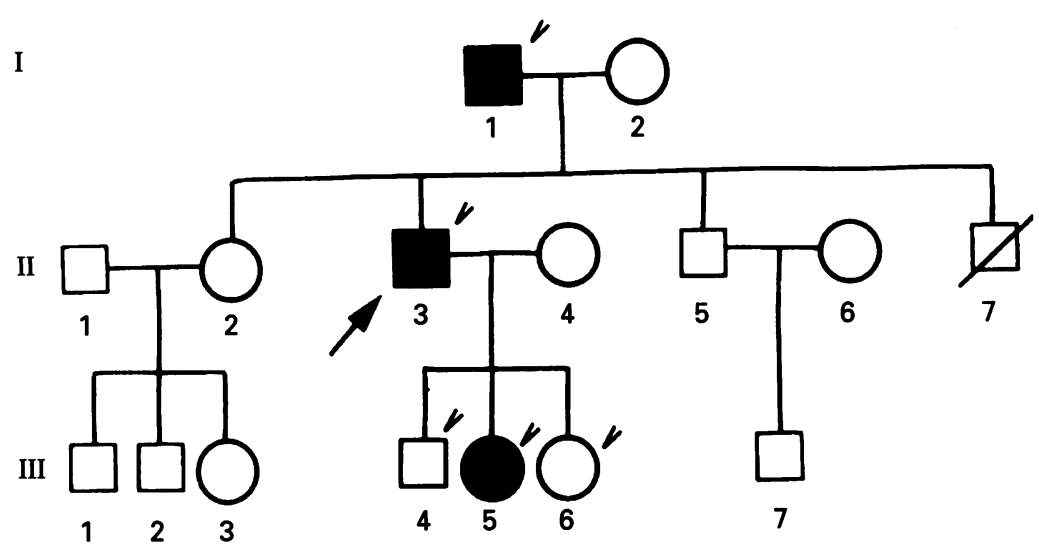

Figure 1 Family pedigree. Arrow indicates proband. ' $V$ ' indicates family members examined. Solid symbols represent affected members.

angiography, EOG, and rod, cone, and flicker ERGs were not performed.

Case 3

The 15-year-old daughter of the proband (III-5; Fig 1) had no complaints about visual acuity, nightblindness, or colour vision. Examination showed her best corrected visual acuity to be $20 / 20$ with a $-1.0 \mathrm{D}$ sphere in both eyes. Slit-lamp examination revealed no abnormal findings in the anterior segments, lens, or vitreous. Ophthalmoscopic and fluorescein angiographic examination showed normal disc, vessels, and retina, including both maculas. Colour vision findings tested by Ishihara plate and panel D-15 were normal. However, the dark adapted bright flash ERG was negatively shaped, with a normal a-wave (Table 1 and Fig 4). The amplitude of the rod b-wave was reduced and showed a delayed implicit time (Table 2 and Fig 4). The amplitude of the cone ERG also was reduced, although the $30 \mathrm{~Hz}$ red flicker ERG showed a normal response (Table 2 and Fig 4). Visual field tested by Humphrey perimetry (program 10-2) was normal (Fig 6). An EOG was not done. Her general physical condition was good.

\section{Discussion}

In this pedigree, we examined five people of three generations in one family and found three affected members (Fig 1). The conditions in our patients were progressive, and their family history showed the inheritance pattern of the disease to be autosomal dominant. None of the patients had a history of taking chloroquine, which can induce bull's eye maculopathy. ${ }^{14}$ Patients with early stages of Batten's disease may have both bull's eye maculopathy and a reduced b-wave response. ${ }^{5}$ Our patients, however, had none of the neurologic abnormalities noted in Batten's disease.

Stargardt's disease (fundus flavimaculatus) reportedly shows macular degeneration including bull's eye maculopathy and a dark choroid by fluorescein angiography. ${ }^{15}$ The fluorescein angiograms from our patients showed normal backgrounds. No reports of patients with Stargardt's disease have described negative

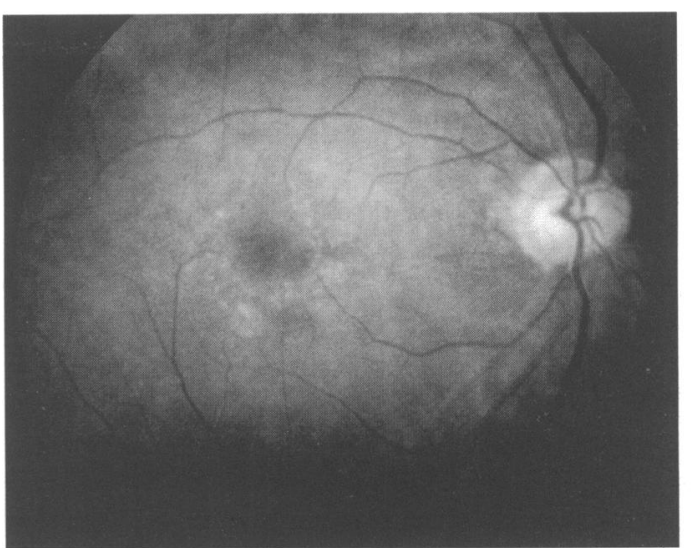

Fig $2 A$

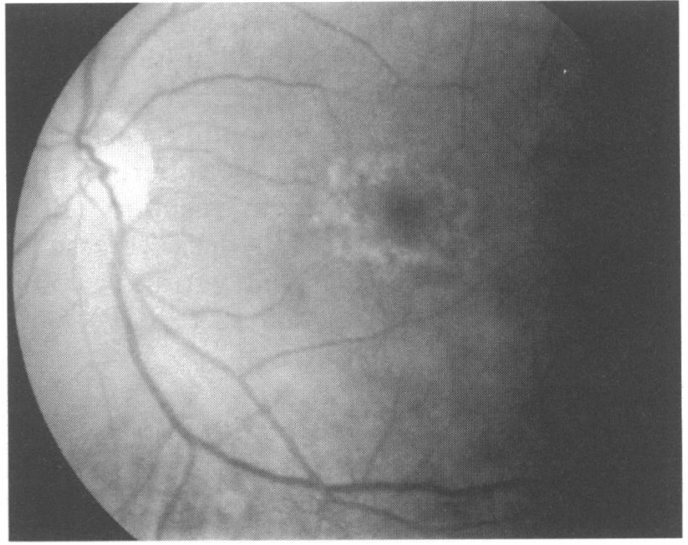

Fig 2B

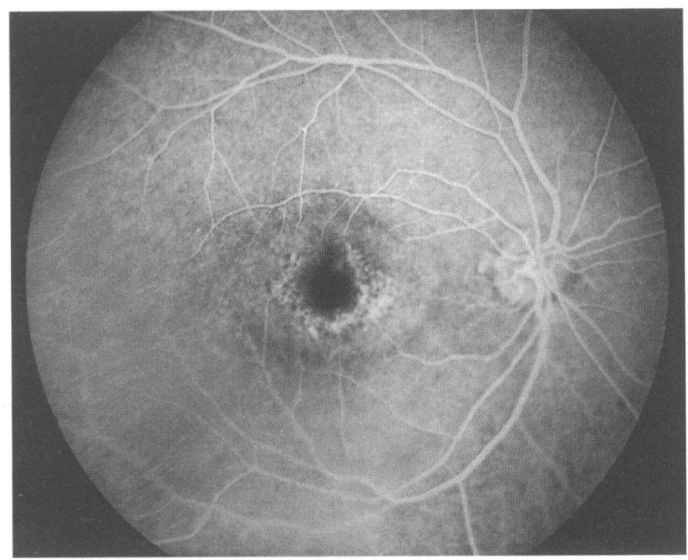

Fig 2C



Fig 2D

Figure 2 Right (A) and left (B) fundus photographs of case 1 show ring-shaped depigmentation around the foveola. Fluorescein angiograms reveal bull's eye maculopathy (C: right eye; D: left eye). 


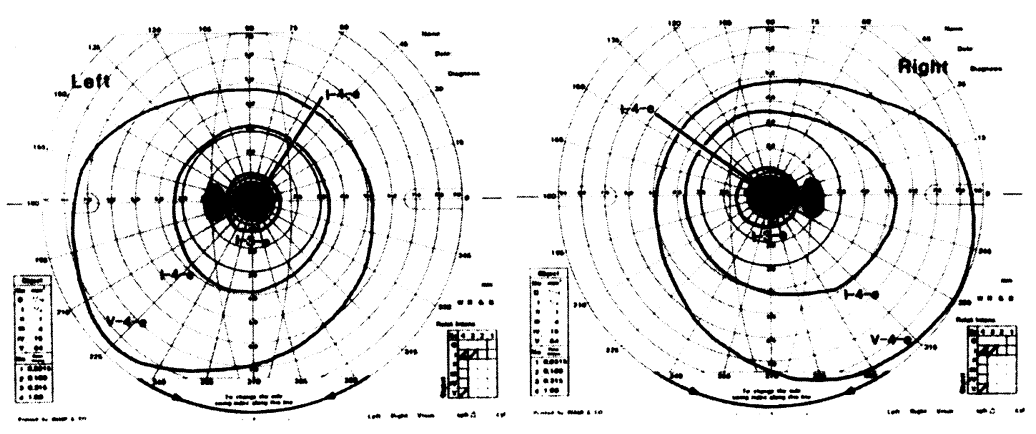

Figure 3 Goldmann perimetric kinetic visual field for case 1 demonstrates central scotoma to I-4-e test target in both eyes.

ERG findings, as found in our patients. Also, the inheritance pattern in Stargardt's disease is usually autosomal recessive, although autosomal dominantly inherited pedigrees have been reported. ${ }^{1617}$ These findings exclude our patients from the diagnosis of Stargardt's disease.

Benign concentric annular macular dystrophy ${ }^{4}$ is characterised by bull's eye maculopathy and autosomal dominant inheritance. Affected patients reveal little visual disturbance, according to Deutman's initial report. No single bright flash ERG was performed in Deutman's patients, so it has been difficult to differentiate those cases from ours. However, a 10 year follow up of the pedigree by Deutman and associates ${ }^{18}$ traced the evolution of benign concentric annular macular dystrophy into a progressive tapetoretinal dystrophy affecting cone and rod function. Peripheral bone corpuscular pigmentation was noted in some patients with progressively decreasing visual acuity and nightblindness. These findings suggested that the disease in our patients is probably not benign concentric annular macular dystrophy.

The fundus findings in our patients would also rule out congenital stationary nightblindness. Patients with congenital stationary nightblindness ${ }^{7}$ have no abnormal fundus findings, although the single bright flash ERG shows a negative shape and the flicker ERG discloses reduced responses. This disorder is non-progressive, and the inheritance is $\mathrm{X}$ linked or autosomal recessive. Sieving and associates ${ }^{19}$ reported an autosomal dominant form of congenital stationary nightblindness associated with Gly-90-Asp rhodopsin mutation. We therefore screened rhodopsin and peripherin/ RDS genes in our affected patients using polymerase chain reaction and single stranded conformation polymorphism analysis. No abnormal rhodopsin or peripherin/RDS genes were, however, found in our patients.

Retinitis pigmentosa was considered as well in the differential diagnosis of our patients. Bull's eye maculopathy is often seen in patients with retinitis pigmentosa. ${ }^{3}$ Cideciyan and Jacobson ${ }^{8}$ recently identified patients who have typical clinical retinitis pigmentosa with a negative ERG. The authors have concluded from the ERG data that dysfunction exists proximal to the photoreceptor terminal lesion and in the photoreceptor outer segment. Vaegan and Graham $^{20}$ have described some patients with cone-rod dystrophy and negative ERG, although precise clinical data were not shown. Our patients had no complaints of nightblindness and normal a-wave amplitudes in a single flash bright ERG. In addition, dark adaptation testing in our proband showed well
Single flash ERG



Scotopic ERG

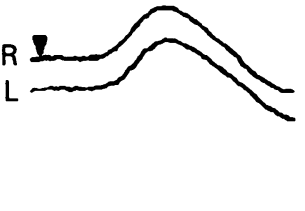

Case 1
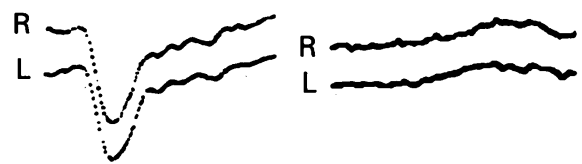

Photopic ERG
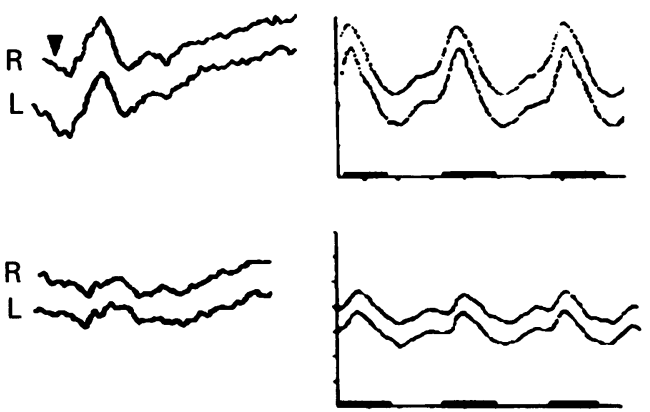

Case 2



Case 3
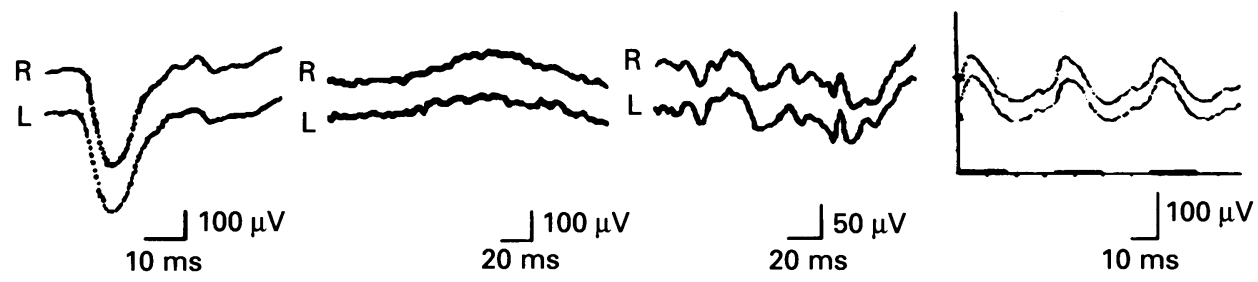

Figure 4 A single flash electroretinogram (ERG) with intense stimulus (a 25 joule bright white flash in cases 1 and 3 and a 40 joule bright flash in case 2) was recorded after 30 minutes of dark adaptation. Compared with a normal control (top), all patients had negative ERGs. The scotopic ERG was first recorded with dim blue light after 30 minutes of dark adaptation. Thereafter, the photopic and ficker ERGs were recorded with red light after 20 minutes of light adaptation. Note the reduced rod and cone b-waves. Low responses also were found in the 30-Hz ficker ERG of case 1 . 



Fig $5 B$

Figure 5 The fundus photographs of case 2 show atrophic lesions in the posterior pole. Choroidal vessels are easily seen (A: right eye; $B$ : left eye).

preserved rod function. Even in the advanced stage, as seen in our proband's father who showed a mildly reduced a-wave, degeneration was restricted to the posterior poles, without pigmentary changes.

Cases of $\mathrm{X}$ linked juvenile retinoschisis ${ }^{6}$ demonstrated a negative ERG and a normal $30 \mathrm{~Hz}$ flicker ERG response. Patients with retinoschisis have a characteristic cystic appearance in the macula with decreased visual acuity from early life. Bull's eye maculopathy, as noted in our patients, does not occur in those with retinoschisis. Miyake and associates $^{3}$ and Kato and Watanabe ${ }^{10}$ recently described patients with bull's eye maculopathy and negative ERG responses. Their patients were male sporadic cases who had had normal

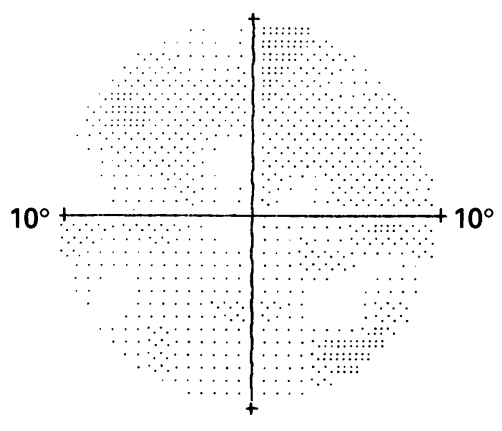

Left

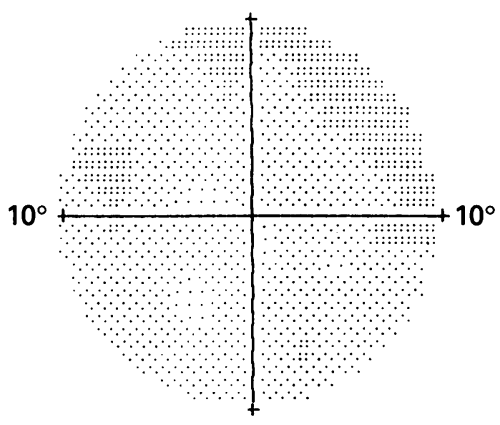

Right
Figure 6 Grey scale representation in decibels of the retinal sensitivity (Humphrey 10-2 program) showed no abnormalities in either eye of case 3. visual acuity that deteriorated with age. The $30 \mathrm{~Hz}$ flicker ERG appeared normal or slightly reduced, which was different from the findings in our cases. In addition, Kato and Watanabe ${ }^{10}$ reported low levels of plasma cyclic GMP in their patients. The plasma level of cyclic GMP was normal in one of our patients, while the cyclic AMP value was slightly lower than normal.

Bull's eye maculopathy is often found in autosomal dominant cone dystrophy. ${ }^{1}$ Disturbed cone ERGs with bull's eye maculopathy and monophasic dark adaptation curve are consistent with the findings in our cases. However, a dark adapted bright flash ERG in our patients showed a negative pattern. The b-wave amplitude was reduced with age. Kellner and Foerster ${ }^{11}$ recently reported on three patients with cone dystrophy and one without symptoms or fundus abnormalities who showed negative photopic ERG. Our cases were autosomal dominantly inherited and had no negative photopic ERG. Therefore, we have concluded that the condition in our cases appears to be a new type of autosomal dominant cone-rod dystrophy with negative ERG, although a new type of autosomal dominant cone dystrophy with negative ERG was not completely ruled out.

Many other retinal disorders ${ }^{21}$ show negative ERG such as Alland Island eye disease, Oguchi disease, infantile Refsum's disease, Goldmann-Favre vitreoretinopathy, ischaemic central retinal vein occlusion, central retinal artery occlusion, retinal toxicity from quinine and vincristine, paraneoplastic syndrome, and optic atrophy. However, these disorders are easily ruled out by their hereditary pattern, fundus findings, and electrophysiological findings.

Single bright flash ERG is thought to become negative when the inner layer of the neuroretina is affected primarily as $\mathrm{X}$ linked retinoschisis ${ }^{6}$ and congenital stationary nightblindness. ${ }^{7}$ An abnormality of neuronal transmission is suggested in congenital stationary nightblindness, while Müller cell abnormalities are thought to occur in $\mathrm{X}$ linked retinoschisis. The deep negative response in $X$ linked retinoschisis can result in a reduced scotopic blue response, although rod function is only slightly damaged. Oscillatory potentials are always abnormal. 62223 Our patients had macular dystrophy, negative ERG with abnormal oscillatory potentials, and reduced rod ERG without nightblindness. These clinical findings appeared to be more similar to ones observed in $\mathrm{X}$ linked retinoschisis than in congenital stationary nightblindness. Therefore, Müller cell abnormalities may be more likely in the conditions of our patients than abnormalities of neuronal transmission, although the precise mechanism remains unknown.

This study was supported in part by grant in aid for Scientific Research from the Ministry of Education, Science and Culture Japan.

1 Krill AE, Deutman AF. Dominant macular degenerations. The cone dystrophies. Am $₹$ Ophthalmol 1972; 73: 352-6. Deutman AF. The hereditary dystrophies of the posterior pole of the eye. Assen: Van Gorcum and Co, 1971: 100-71. 
3 Fishman GA, Fishman M, Maggiano J. Macular lesions associated with retinitis pigmentosa. Arch Ophthalmol 1971; 95: 798-802.

4 Deutman AF. Benign concentric annular macular dystrophy. Am f Ophthalmol 1974; 78: 384-96.

5 Spalton DJ, Taylor DSI, Sanders MD. Juvenile Batten's disease: an ophthalmological assessment of 26 patients. Br f Ophthalmol 1980; 64: 726-32.

6 Deutman AF. X-linked juvenile retinoschisis. In: Krill A ed. Hereditary retinal and choroidal diseases. Vol II. Clinical ed. Hereditary retinal and choroidal diseases. Vol II. Clinical
characteristics. Hagerstown: Harper \& Row, 1976: characteristics.

7 Miyake Y, Yagasaki K, Horiguchi M, Kawase Y, Kanda T. Congenital stationary night blindness with negative electroretinogram. A new classification. Arch Ophthalmol 1986; 104: 1013-20.

8 Cideciyan AV, Jacobson SG. Negative electroretinograms in retinitis pigmentosa. Invest Ophthalmol Vis Sci 1993; 34: 3253-64.

9 Miyake Y, Shiroyama N, Horiguchi M, Saito A, Yagasaki K. Bull's-eye maculopathy and negative Yagasaki K. Bull's-eye maculopathy and negative

10 Kato M, Watanabe I. Bull's-eye maculopathy, negative electroretinogram and low plasma cyclic guanosine monophosphate level. A report of two cases. Doc Ophthalmol 1990; 75: 23-32.

11 Kellner U, Foerster MH. Cone dystrophies with negative photopic electroretinogram. Br 7 Ophthalmol 1993; 77: 404-9.

12 Tahara K, Matsumoto H, Kawano M, Kawabata E, Otori T. Clinical experience with a new ERG contact lens for recording $30 \mathrm{~Hz}$ flicker ERG. F Eye (尹pn) 1989; 6: recording

13 Kikawa E, Nakazawa $M$, Chida $Y$, Shiono T, Tamai $M$ A novel mutation (Asp244Lys) in the peripherin/RDS gene causing autosomal dominant retinitis pigmentosa associated with bull's-eye maculopathy detected by nonradioisotopic SSCP. Genomics 1994; 20: 137-9.
14 Kearns TP, Hollenhorst RW. Chloroquine retinopathy. Evaluation of fluorescein angiography. Arch Ophthalmol 1966; 76: 378-84.

15 Fish G, Gery R, Shemi KS, Bird AC. The dark choroid in posterior retinal dystrophies. Br f Ophthalmol 1981; 65: 359-63.

16 Merin S, Landau J. Abnormal findings in relatives of patients with juvenile hereditary macular degeneration patients with juvenile hereditary macular degeneration
(Stargardt's disease). Ophthalmologica 1970; 161:

17 Krill AE. Fundus flavimaculatus. In: Krill AE, ed. Hereditary retinal and choroidal diseases. Vol II. Clinical characteristics. Hagerstown, Maryland: Harper \& Row, 1977: 741-87.

18 van den Biesen PR, Deutman AF, Pinckers AJLG. Evolution of benign concentric annular macular dystrophy. Am f Ophthalmol 1985; 100: 73-8.

19 Sieving PA, Richards JE, Bingham EL, Naarendorp F. Dominant congenital complete nyctalopia and Gly90Asp rhodopsin mutation. Invest Ophthalmol Vis Sci 1992; 33: 1397 .

20 Vaegan, Graham S. Well preserved photoreceptor and neural function in early cone/rod dystrophies: an initial abnormality in inner retina? Invest Ophthalmol Vis Sci 1991; 32 (suppl): 1138.

21 Heckenlively JR, Weleber RG, Arden GB. Testing levels of the visual system. In: Heckenlively JR, Arden GB, eds. Principles and practice of clinical electrophysiolog of vision. St Louis: Mosby-Year Book, 1991: 485-93.

22 Hirose T, Wolf E, Haran A. Electrophysiological and psychophysical studies in congenital retinoschisis of X-linked recessive inheritance. Doc Ophthalmol Proc Series 1977; 13: 173-84.

23 Peachey NS, Fishman GA, Derlacki DJ, Grigell MG. Psychophysical and electroretinographic findings in $\mathrm{X}$-linked juvenile retinoschisis. Arch Ophthalmol 1987 105: 513-6. 